Published in Adv Protein Chem Struct Biol. 2019;115:1-19. doi:

10.1016/bs.apcsb.2018.10.003

\title{
DNA Repair by Photolyases
}

Ibrahim Halil Kavakli ${ }^{1,2^{*}}$ and Nuri Ozturk ${ }^{3}$, Seref Gul ${ }^{1}$

${ }^{1}$ Koc University Departments of Chemical and Biological Engineering and ${ }^{2}$ Molecular

Biology and Genetics, Sariyer, Istanbul, Turkey

${ }^{3}$ Gebze Technical University, Department of Molecular Biology and Genetics, Gebze, Kocaeli, Turkey

${ }^{*}$ Corresponding author

e-mail: hkavakli@ku.edu.tr (Ibrahim Halil Kavakli) 


\begin{abstract}
Photolyases belong to the cryptochrome/photolyase protein family (CPF) which perform different functions such as DNA repair, circadian photoreceptor, and transcriptional regulation. Photolyase protein is a DNA repair enzyme that repairs UV-induced DNA damages of cyclobutane pyrimidine dimer (CPD) and pyrimidine-pyrimidone (6-4) photoproducts using blue-light as an energy source. This enzyme is a flavoprotein containing two chromophores: flavin adenine dinucleotide (FAD) as a cofactor and a photoantenna such as methenyltetrahydrofolate (MTHF). The FAD is essential for catalysis DNA repair. MTHF absorbs photons from the blue light spectrum and transfers energy to FAD to increase the repair efficiency of the enzyme. Phylogenetic analysis in which amino acid sequences of several hundreds of CPF members are used suggests that they form more classes than we have considered so far. In this chapter, we discussed structure-functions and reaction mechanisms of different classes of photolyases.
\end{abstract}




\section{Table of Contents}

1. Introduction $\quad 4$

2. Cryptochrome/Photolyase protein family 5

2.1 Cofactors of the Photolyases 9

2.2 The crystal structure of the photolyase 12

2.3 Reaction Mechanism of DNA repair Mediated by CPD Photolyase 16

2.4 Reaction mechanisms of DNA repair mediated by (6-4) and other classes of the photolyases 19

3. Conclusion 23

4. Acknowledgment 24

$\begin{array}{lr}\text { 5.References } & 24\end{array}$ 


\section{Introduction}

DNA is the source of genetic information in all living cells. Therefore, its stability and integrity are crucial to life. Considering its chemical nature it can undergo several different modifications (induced by factors such as oxidative damage, ultraviolet and other types of radiation, and hydrolytic damage), which can cause alterations in its composition. In addition, the DNA sequence can also change during DNA replication despite proofreading activity of the DNA polymerases. Modified/altered DNA structure needs to be corrected in either case to prevent mutations and genomic instability of the cells. If these modifications and base changes permanently stay in the genome of organisms they can lead to the development of cancers depending on the nature of mutations. Therefore, cells possess different types of the DNA repair mechanisms to minimize mutagenic changes. There are mainly two types of DNA repair mechanisms, which are single strand (ss)- and double strand (ds)-DNA damage repair. The ssDNA damage repair mechanisms are further divided into direct reversal repair, nucleotide excision repair, base excision repair, and mismatch repair while dsDNA damage repair mechanisms are divided into homologous recombination and non-homologous end joining repairs. Among these, direct reversal repair mechanism doesn't require template information and corrects damages by simply reversing those using DNA repair enzymes. Three major direct repair mechanisms are mediated by photolyases, $O^{6}$-alkylguanineDNA alkyltransferases AGTs, and the AlkB family dioxygenases, which reverse UV light-induced photolesions, $O$-alkylated DNA damage, and $N$-alkylated base adducts, respectively (Yi \& He, 2013). In this chapter, we will highlight structure-functions and reaction mechanisms mediated by photolyases. 


\section{Cryptochrome/Photolyase protein family}

The quality and quantity of ultraviolet (UV) radiation reaching the earth's surface from the sun depends on the transmission properties of the atmosphere, which has changed over the course of time as a result of human activity. For example, ozone layer thickness has been declining for a century, which causes more UV to reach the earth's surface (Bais et al., 2015). UV irradiation induces DNA damage in the form of cyclobutane pyrimidine dimers (Pyr $<>\mathrm{Pyr}, \mathrm{CPD}, \sim 80-90 \%$ ) and pyrimidine-pyrimidone (6-4) photoproducts (Pyr [6-4] Pyr, 10-20\%) in organisms (Fig. 1) (Menck, 2002; Thompson \& Sancar, 2002). This damage is reversed by DNA photolyases using bluelight (350 -500 nm) as an energy source (Fig. 1) in some organisms and by the nucleotide excision repair in others including those with photolyases (A. Sancar, 2003). Molecular evolutionary analysis, genetic and biochemical studies with photolyase-related genes, isolated from several hundred organisms throughout years, revealed that this family forms different classes with different functions such as DNA repair, photoreceptor, and transcriptional regulators. Collectively these genes are named as cryptochrome/photolyase protein family (CPF). Several reviews on the CPF have been published in the past years (Chaves et al., 2006; Kavakli et al., 2017; Konig, Juhas, Jager, Kottke, \& Buchel, 2017; Michael, Fribourgh, Van Gelder, \& Partch, 2017; Ozturk, 2017). Phylogenetic analyses of more than 250 photolyase/cryptochrome family members have revealed several major classes (Fig. 2) (Asimgil \& Kavakli, 2012; Kavakli et al., 2017; Ozturk, 2017; Ozturk et al., 2008). These include Class I CPD photolyase, Class II CPD photolyase, Class III CPD photolyase, (6-4) photolyase and single-strand specific DNA photolyases (previously known as DASH-CRY). Class I and (6-4) photolyases with high 
similarity at structural levels (Thompson \& Sancar, 2002; M. Zhang, Wang, \& Zhong, 2017) repair Pyr $<>$ Pyr dimers and Pyr[6-4]Pyr photoproducts, respectively (A. Sancar, 2003, 2016).

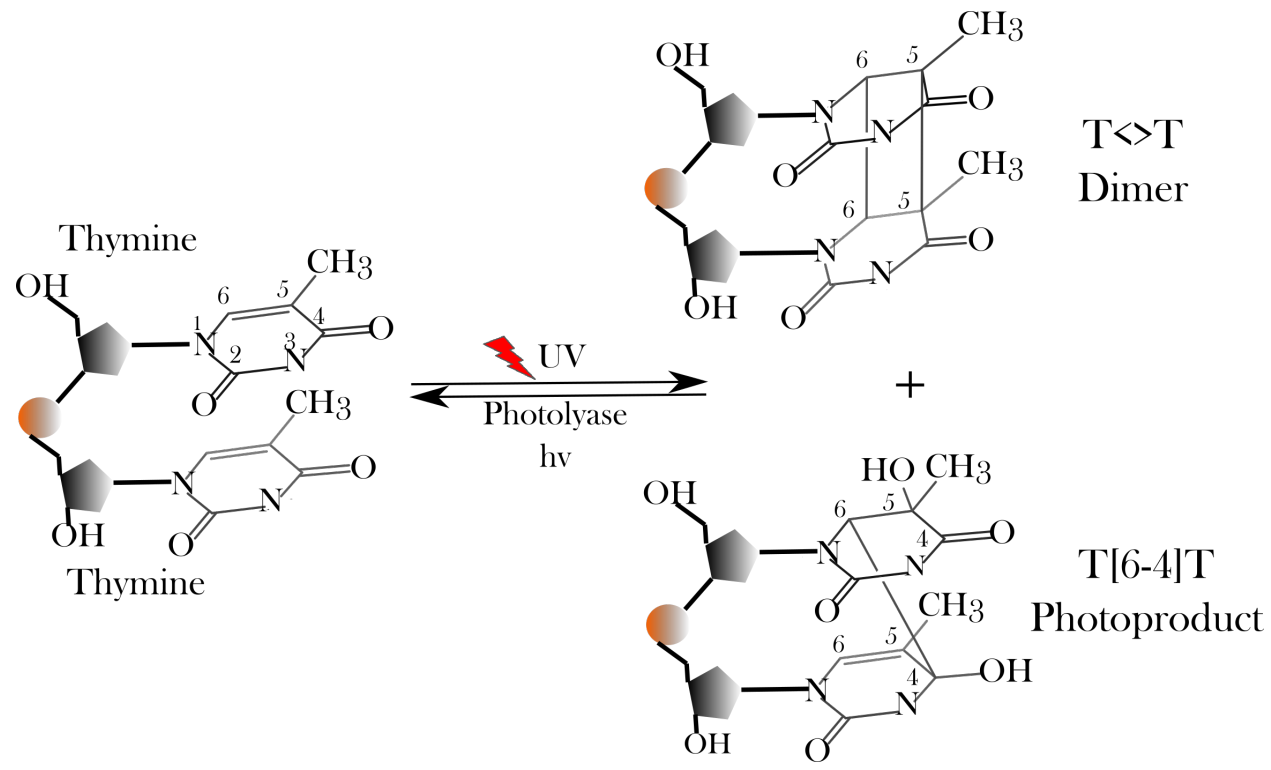

Fig. 1: Ultraviolet (UV) induces DNA damages and causes the formation of photoproducts. Damages can be repaired by photolyases utilizing the blue light as an energy source. For the sake of simplicity, DNA is shown as single-stranded. 


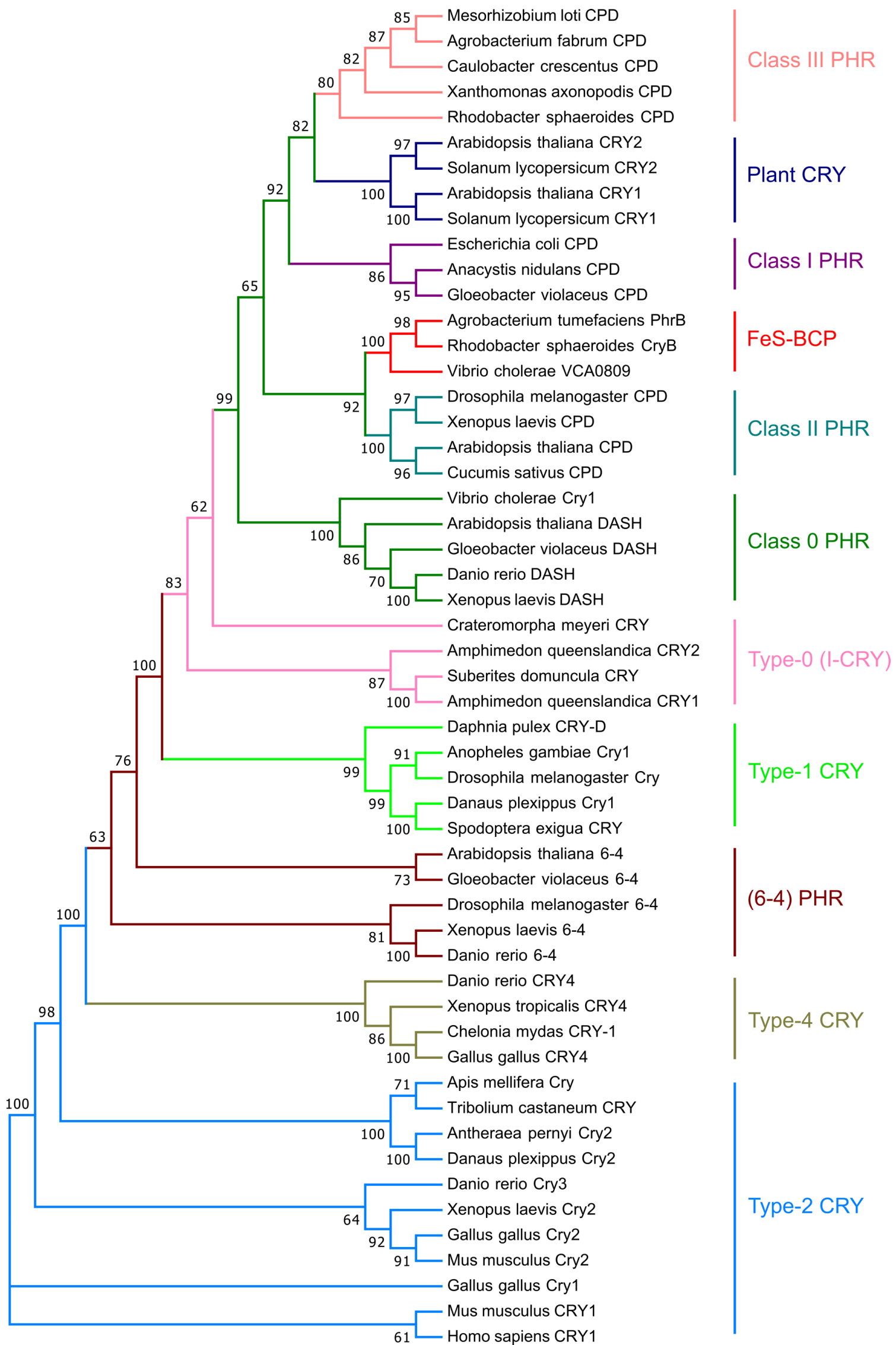


Fig. 2: Phylogenetic tree of the cryptochrome/photolyase family. The unrooted phylogenetic tree of CPF member was inferred using the neighbor-joining method. The optimal tree with the sum of branch lengths $=10.044$

The (6-4) photolyases have previously been considered as only one type and exist only in eukaryotes. However, a study with a soil born gram-negative bacteria Agrobacterium tumefaciens indicated the presence of a new type of (6-4) photolyase gene named Phr B while class III photolyase gene was named as PhrA. PhrB belongs to a new class named iron-sulfur bacterial cryptochromes and photolyases (FeS-BCP) (F. Zhang, Scheerer, Oberpichler, Lamparter, \& Krauss, 2013). We have to note that this nomenclature of PhrA and B should not be confused with the historical nomenclature used during early identification of photoreactivation in E. coli. Two genetic loci (phrA and $\mathrm{phrB}$ ), which are responsible for photoreactivation, have been proposed, however, it was shown that there is only one photolyase in E. coli (Husain \& Sancar, 1987). This nomenclature was left, and abbreviation of phr (not phr A or B) in E. coli was exchanged with CPD photolyase. The group of FeS-BCPs shows the largest phylogenetic distance to the other groups of the CPF. In addition to these unusual (6-4) photolyases, another (6-4) photolyase has been suggested to exist in Gloeobacter violaceus (Ozturk et al., 2007). Even though FeS-BCP exists only in prokaryotes, the presence of classical (6-4) photolyase in Gloeobacter does not allow us to classify (6-4) photolyases as prokaryotic or eukaryotic. It might be the right time to consider (6-4) photolyases as Class I (Eukaryotic), Class II (Fe-S BCP) and Class III (Gloeobacter (6-4) photolyase-like).

The CRY-DASHs were found based on phylogenetic and structural analyses of proteins found in Drosophila melanogaster, Arabidopsis thaliana, Synechocystis and $\underline{H}$ omo sapiens (Brudler et al., 2003). CRY-DASHs were originally 
thought to be a class of CRYs because of the lack of repair activity on dsDNA and failure of bacterial complementation assay in photolyase deficient E.coli (Hitomi et al., 2000; Worthington, Kavakli, Berrocal-Tito, Bondo, \& Sancar, 2003). However, a subsequent study with a CRY-DASH from Vibrio cholerae (VcCRY1) showed photolyase activity, where VcCRY1 specifically repairs ssDNA but not dsDNA (Selby \& Sancar, 2006). It is interesting to note that these types of proteins found in multiple copies form in algae (Asimgil \& Kavakli, 2012; Chaves et al., 2011) and their functions are reviewed in (Noordally \& Millar, 2015).

\subsection{Cofactors of the Photolyases}

Photolyases (PLs) are monomeric proteins with a molecular mass range from 50 to $61 \mathrm{kDa}$. They are present in bacteria, plants, fishes, birds, fungi and marsupial animals (Menck, 2002). PLs are associated with two chromophores flavin adenine dinucleotide (FAD) (Fig. 3A) and methenyltetrahydrofolate (MTHF) (Fig. 3B) or 8hydroxy-7,8-didemethyl-5-deazariboflavin (8-HDF) (Fig. 3C) (G. B. Sancar \& Sancar, 1987). Studies with A. tumefaciens Fe-S BCP PL revealed the presences of the new cofactors: Fe-S cluster, and 6,7-dimethyl-8-ribityllumazine (DMRL). DMRL is an antenna chromophore (Fig. 3D) (Oberpichler et al., 2011; F. Zhang et al., 2013). Finally, it has been shown that Thermus thermophiles photolyase possess flavin mononuclotide (FMN) as a second chromophore (Fig. 3A) (Ueda, Kato, Kuramitsu, Terasawa, \& Shimada, 2005).

A)

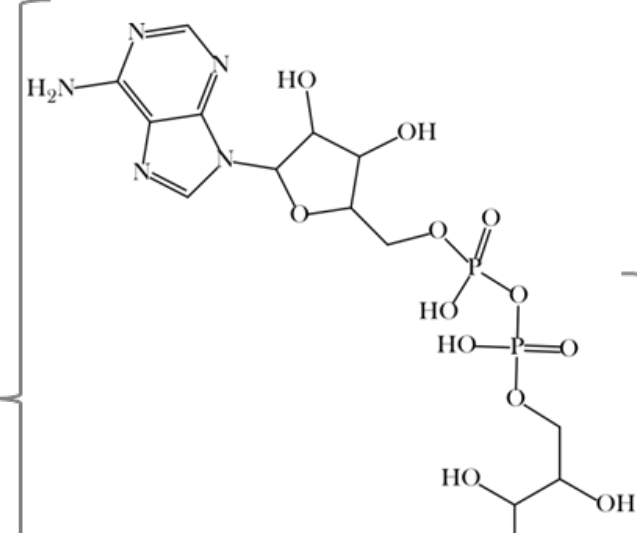

B)

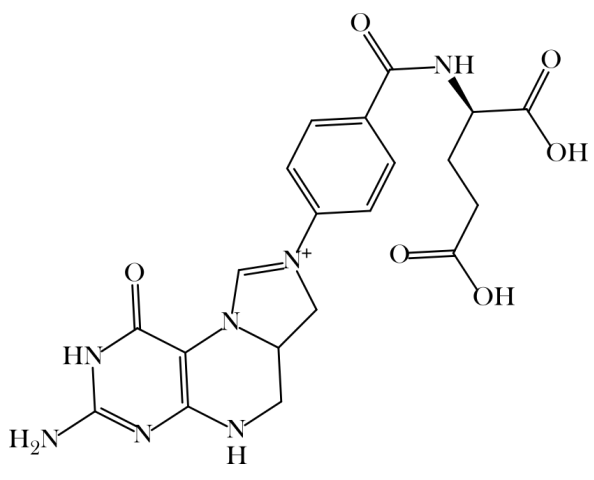


C)

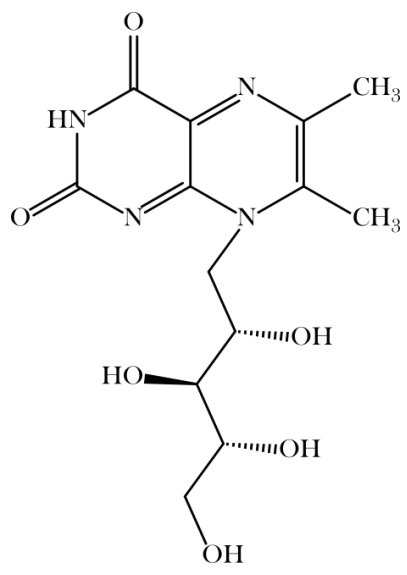

D)

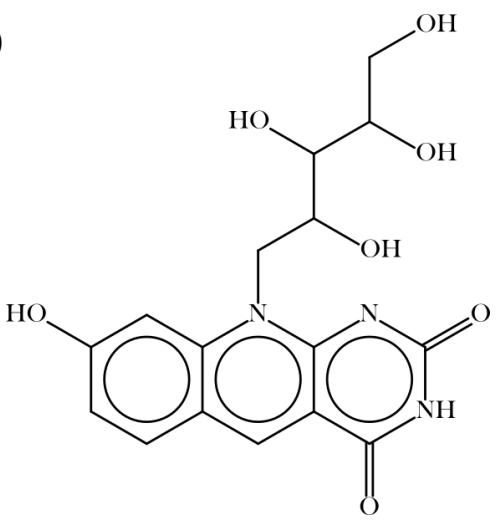

Fig. 3: Structure of chromophores of photolyases. A) flavin adenine dinucleotide (FAD), flavin mononucleotide (FMN) B) methenyltetrahydrofolate MTHF, C) 8-hydroxy-7,8didemethyl-5-deazariboflavin (8-HDF), D) 6,7-dimethyl-8-ribityllumazine (DMRL)

Endogenous level of photolyase is quite low, around 15-16 molecules per E.coli cell (Harm, Harm.H., \& Rupert, 1968; Kavakli \& Sancar, 2004). To obtain a sufficient amount of PL for its biophysical and biochemical characterization, recombinant DNA technologies were employed in E.coli cells. E.coli phr was the first over-expressed PL gene. Analysis of purified E.coli photolyase protein (EcPL) indicated both apoenzyme and FAD are in 1:1 ratio (A. Sancar \& Sancar, 1984). FAD is strongly bound to the protein in a non-covalent manner. FAD is the required cofactor for the catalysis of the 
PLs and its binding to damaged DNA (substrate) (Jorns, Baldwin, Sancar, \& Sancar, 1987; G. B. Sancar et al., 1987). There are four different redox states of FAD in photolyases after the purification: oxidized (FAD), anionic semiquinone (FAD*-), neutral semiquinone $\left(\mathrm{FADH}^{*}\right)$, and anionic hydroquinone $\left(\mathrm{FADH}^{-}\right)$. Each state of $\mathrm{FAD}$ can be differentiated by absorption spectrum. Purified EcPL exhibits different peaks: the peak at $380 \mathrm{~nm}$ stemming mostly from MTHF and $480 \mathrm{~nm}, 580 \mathrm{~nm}$, and $625 \mathrm{~nm}$ peaks comes from blue-neutral radical form FAD (neutral radical form of FAD: FADH•) (Fig. 4). $\mathrm{FADH}^{-}$is the active form for the DNA repair in CPD photolyases during the course of the catalysis (Kim, Sancar, Essenmacher, \& Babcock, 1993). Different oxidized forms of FAD in the PLs can be reduced either photochemically or with dithionite treatment.<smiles></smiles>

$\mathrm{FADH} \cdot, 480 \mathrm{~nm}, 580 \mathrm{~nm}, 625 \mathrm{~nm}$<smiles>[Y]N1c2cc(C)c(C)cc2Nc2c1[n-]c(=O)[nH]c2=O</smiles>

$\mathrm{FADH}^{-}, 366 \mathrm{~nm}$

Fig. 4: Different oxidation states of the flavin adenine dinucleotide (FAD) and their characteristic peak values in the absorption spectra. FAD accepts one electron and one proton, both of which appear in the flavin ring system (isoalloxazine), the semiquinone, stable radical, $\mathrm{FADH} \bullet$, forms. When $\mathrm{FADH} \bullet$ accepts one electron, fully reduced $\mathrm{FADH}^{-}$ forms.

Unlike FAD, MTHF with varying number of glutamate is loosely attached to PL. It can easily dissociate from the PL during the purification of the enzyme. MTHF increases the rate of DNA repair 10-100-fold rather than affecting catalysis or substrate binding (Payne \& Sancar, 1990). This cofactor exhibits maximum light absorbance at $360 \mathrm{~nm}$ for the EcPL. The interaction between the MTHF and proteins can alter the 
maximum absorbance value (Eker, Yajima, \& Yasui, 1994; A. Sancar, 2003). The 5,10methenyl bridge of the MTHF is heat labile. Therefore heat can break the bridge and MTHF cannot absorb light near-UV absorption at $360 \mathrm{~nm}$. Like MTHF, 8-HDF is shown to act as photo antenna in Anacystis nidulans (AnPL) exhibiting an absorption maximum at $440 \mathrm{~nm}$ (A. Sancar, 2003).

In addition to $\mathrm{FAD}$, the $\mathrm{Fe}-\mathrm{S} \mathrm{BCP}$ protein family has different cofactors compared with other PLs. These are the Fe-S cluster and DMRL (Fig. 3C). Regarding the role of [4Fe-4S] in Fe-S BCP its function is yet to be shown. However, mutagenesis of Cys residues coordinating the Fe-S cluster suggested that this domain is required for the stability of the Fe-S BCP (Graf et al., 2015; M. Zhang et al., 2017) and Cys residues can play a role in electron transfer (White \& Dillingham, 2012). DMRL is the second antenna shown by crystal structure and HPLC studies of the Fe-S BCP. In fact, the I51W mutation in the Fe-S BCP decreased the photoreduction, and the DNA repair capacity. Crystal structure of I51 W mutant Fe-S BCP showed that the DMRL binding pocket is mostly occupied by the tryptophan residue (W51). Therefore, DMRL can not bind to the I51W mutant Fe-Ss BCP (F. Zhang et al., 2017). Hence, role of DMRL has been proposed as to transfer energy to FAD during the course of catalysis.

\subsection{The crystal structure of the photolyase}

The crystal structures of the PLs from different organisms are solved via X-ray crystallography (Fujihashi et al., 2007; Hitomi et al., 2009; Kiontke et al., 2011; Komori et al., 2001; Park, Kim, Sancar, \& Deisenhofer, 1995; Scheerer et al., 2015; Tamada et al., 1997; F. Zhang et al., 2017; F. Zhang et al., 2013). Comparison of the structures revealed that PLs have two-distinct domains connected by a loop having different amino acid residues depending on the organismal source (Fig. 5). The first domain called the N- 
terminal $\alpha / \beta$ domain consists of five $\beta$-sheets and five $\alpha$-helices, which is a typical dinucleotide-binding domain. The second domain, called C-terminal $\alpha$-helical domain, consists of 14-helices and has a cavity where the catalytic cofactor FAD is located. The FAD is commonly present in U-shaped conformation by 14 interacting amino acid residues (A. Sancar, 2008). The majority of amino acids interacting with FAD are conserved at the exact same positions between PLs with some exceptions. For example, 12 FAD interacting amino acids are conserved between EcPL, AnPL and Thermus thermophiles PLs (TtPL) with the exception of Phe-307 and Val-346 of EcPL, which are replaced by tryptophan and alanine respectively in TtPL (Komori et al., 2001). Additionally, the $\mathrm{N} 5$ atom of the isoalloxazine moiety is protonated and hydrogen bonded to an Asn-378 residue in TtPL, which further stabilizes the FAD interaction with apoprotein (Xu et al., 2008). 


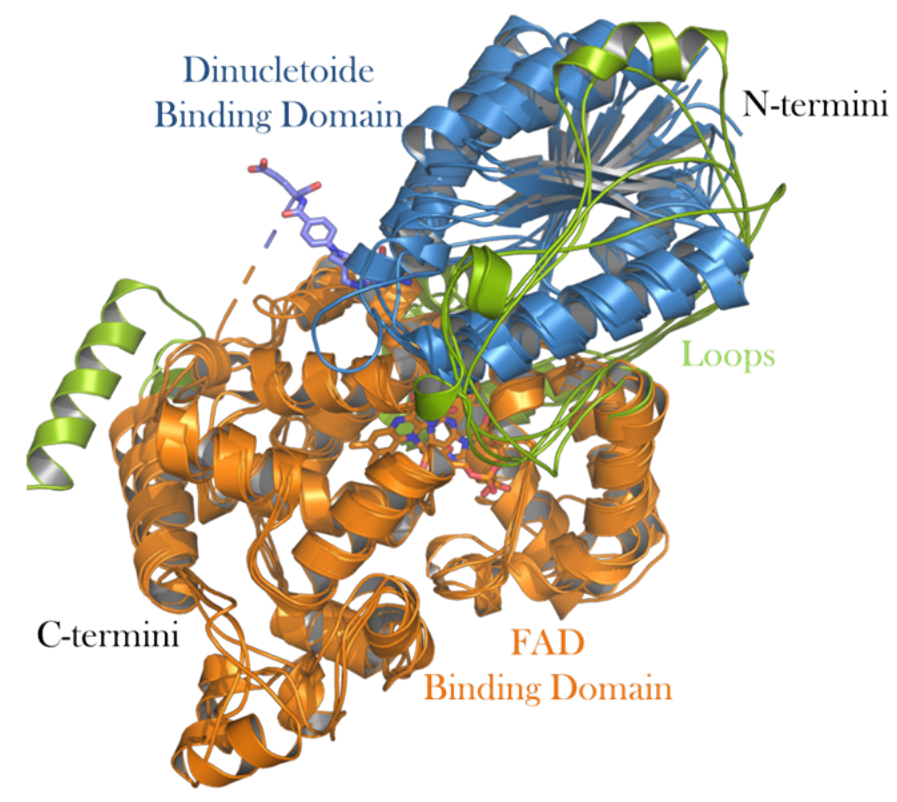

Fig. 5: Superimposition of CPD photolyases of Escherichia coli, Thermus thermophilus, Anacystis nidulans and (6-4) photolyase of Arabidopsis thaliana. Blue color indicates the $\mathrm{N}$-terminal $\alpha / \beta$ domain while orange color indicates the $\mathrm{C}$-terminal $\alpha$-helical domain.

The second light harvesting MTHF cofactor is located in the cleft between two domains of the PL. The distance from MTHF to FAD is calculated as $16.8 \AA$ in EcPL (Park et al., 1995). AnPL has an additional space in the interior part between the clefts where it accommodates MTHF (Tamada et al., 1997). PLs specifically bind either to ssDNA or dsDNA containing Pyr $<>$ Pyr damage with the specific binding constant $\left(\mathrm{K}_{\mathrm{d}}\right)$ of the $10^{-9} \mathrm{M}$ (Husain \& Sancar, 1987). Analysis of the solvent accessible surface of EcPL indicates that FAD can interact Pyr $<>$ Pyr through the hole (Fig. 6A) (Park et al., 1995). Surrounding regions of the hole are rich with positively charged amino acid residues indicated by the blue color. In fact replacement of the amino acids in the blue region result in the change the affinity of the EcPL to the substrate (Baer \& Sancar, 1993). 

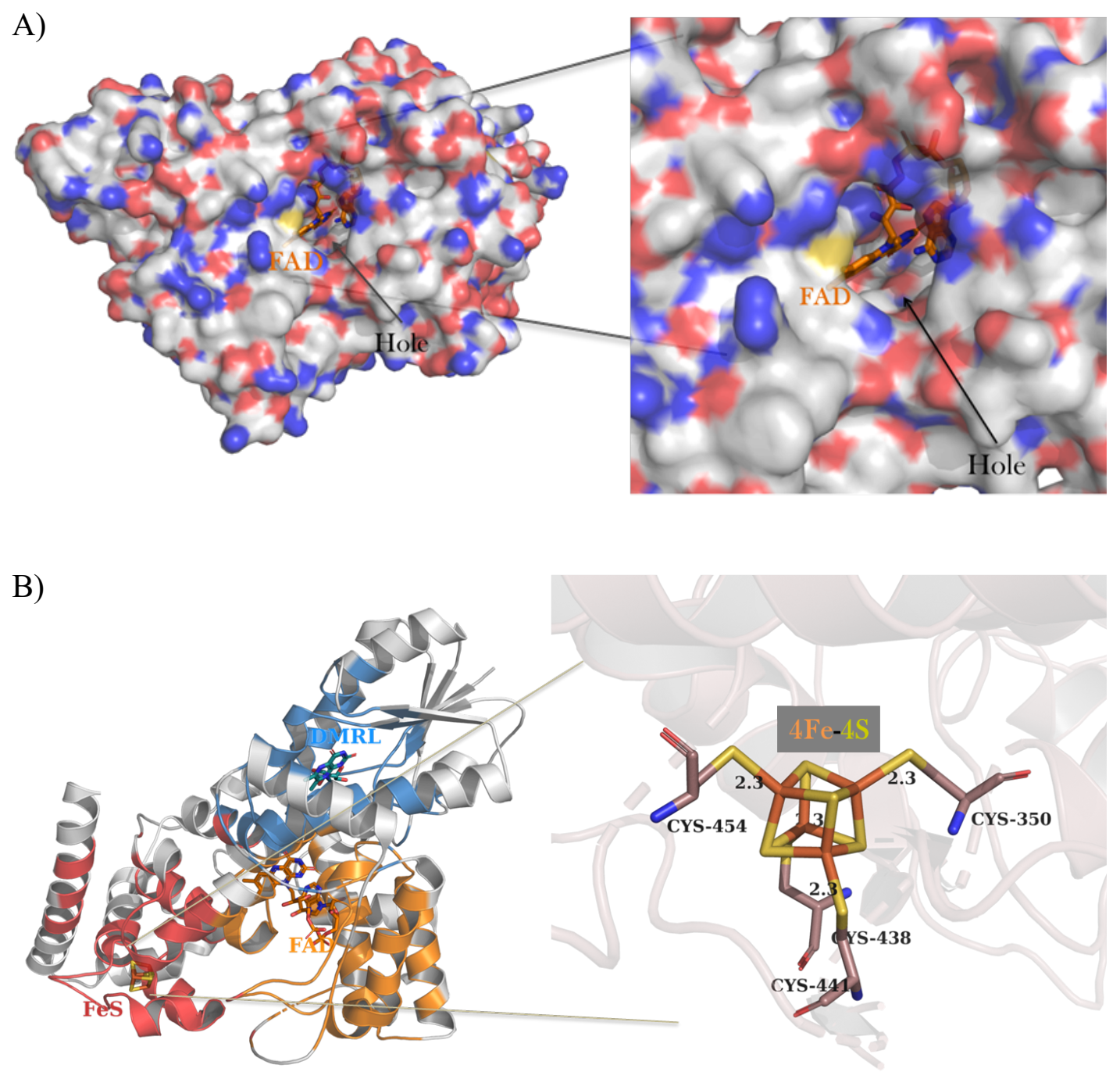

Fig. 6: A) Surface contour image indicates FAD binding region and surface exposed amino acids of E.coli photolyase (PDB ID: 1DNP). Blue color represents amino acids with basic groups while red color represents amino acids with acidic groups. Carbon atoms of FAD are shown in orange. B) (6-4) photolyase from Agrobacterium tumefaciens (PDB ID: 4DJA) and cofactor binding pockets. There are 4-cysteines interacting with the $4 \mathrm{Fe}-4 \mathrm{~S}$ cluster. Fe atoms are shown in orange and Sulphur atoms in yellow.

The crystal structures of the ssDNA repair enzymes of Arabidopsis thaliana Cryptochrome 3 (AtCRY3) and Synechocystis CRY (also called as CRY-DASHs) have also been determined (Brudler et al., 2003; Huang et al., 2006; Klar, Pokorny, Moldt, Batschauer, \& Essen, 2007). Analysis showed that the overall structures of the CRY DASHs are very similar to class I EcPL with some differences. One main difference is 
that AtCRY3 DNA binding region is more polar compared to class I PLs. Although the substrate-binding regions of both classes of enzymes are similar, CRY-DASH has a weak affinity towards damaged or undamaged dsDNA (Kizilel et al., 2012).

The crystal structure of Fe-S BCP from A. tumefaciens is solved with a resolution of 1.45 $\AA$ (F. Zhang et al., 2013). The comparison of this structure with other PLs revealed a very similar 3D shape with root-mean-square difference (RMSD) values range from $2.8 \AA$ and 3.5 A. The FAD is present in a U-shaped conformation and stabilized by hydrogen bonding interactions with the side chain of Arg369 and the carbonyl group of Tyr391 in Fe-S BCP. The analysis of the second chromophore-binding site suggested that only DMRL could fit with the electron density map and its presence is confirmed by HPLC. Unlike EcPL DNA binding region, where one side has hydrophobic residues and other side has polar residues, the Fe-S BCP substrate binding site is very similar to (6-4) A.thaliana PL (AtPL) where His-His-X-X-Arg motif (Hitomi et al., 2001) is conserved in FeS-BCP. The replacement of the conserved His366 in FeS-BCP results in loss of its DNA repair activity (Graf et al., 2015). This is consistent with previous studies where it is shown this particular amino acid is required for DNA repair in (6-4) PLs. The FeSBCP possesses a $[4 \mathrm{Fe}-4 \mathrm{~S}]$ cluster in which $4 \mathrm{Fe}$ atoms are coordinated by 4 Cys residues (Cys-350, Cys 438, Cys441, and Cys454 in Agrobacterium tumefaciens) (Fig. 6B).

\subsection{Reaction Mechanism of DNA repair Mediated by CPD Photolyase}

The reaction mechanism of the Class I CPD photolyase has been revealed using EcPL (A. Sancar, 2003, 2008, 2016). Photolyase binds to the backbone of the damaged DNA strand by ionic interactions. This binding occurs around the cyclobutane dimer in a light-independent manner. Upon binding, PL flips the dimer out into the active site cavity to make contact with the flavin (Mees et al., 2004; Park et al., 1995) which leads to stable 
enzyme-substrate complex (Jordan, Alderfer, Chanderkar, \& Jorns, 1989; Jorns, Sancar, \& Sancar, 1985). Exposure of this complex to light initiates catalysis; the FADH and MTHF photoantenna absorb a photon and MTHF transfers the excitation energy to fully reduce $\mathrm{FADH}^{-}$by Förster dipole-dipole resonance energy transfer to increase the efficiency of the overall DNA repair. Light absorbance generates excited $\mathrm{FADH}^{-*}$ which donates an electron to the $\mathrm{Pyr}<>\mathrm{Pyr}$, where it cleaves the $\mathrm{C}^{-}-\mathrm{C}^{`}{ }^{\prime}$ and $\mathrm{C} 6-\mathrm{C} 6{ }^{\prime}$ bonds of the cyclobutane ring and generates free thymine bases. The enzyme and the product dissociate. The electron is transferred back into $\mathrm{FADH}^{-*}$ to regenerate $\mathrm{FADH}^{-}$, ready for the next round of catalysis (Fig. 7). Photolyase repairs damaged DNA using a mechanism of light-induced electron transfer that does not result in a net change of the redox state of the enzyme (A. Sancar, 2016). This repair is achieved with a high quantum yield of 0.84 . To understand how this is achieved Tan et al. performed femtosecond spectroscopy using EcPL (Tan et al., 2015). Their results revealed the presence of the cyclic electron tunneling mechanism. Unique bended structure of FAD within the EcPL where Adenine and lumi-flavin moieties of FAD come very close together which allows fast direct intramolecular electron transfer from lumi-flavin moiety of four redox states of FAD to the substrate of $\mathrm{Pyr}<>\mathrm{Pyr}$ in 250 ps through direct tunneling (Liu et al., 2011; Liu et al., 2013). 


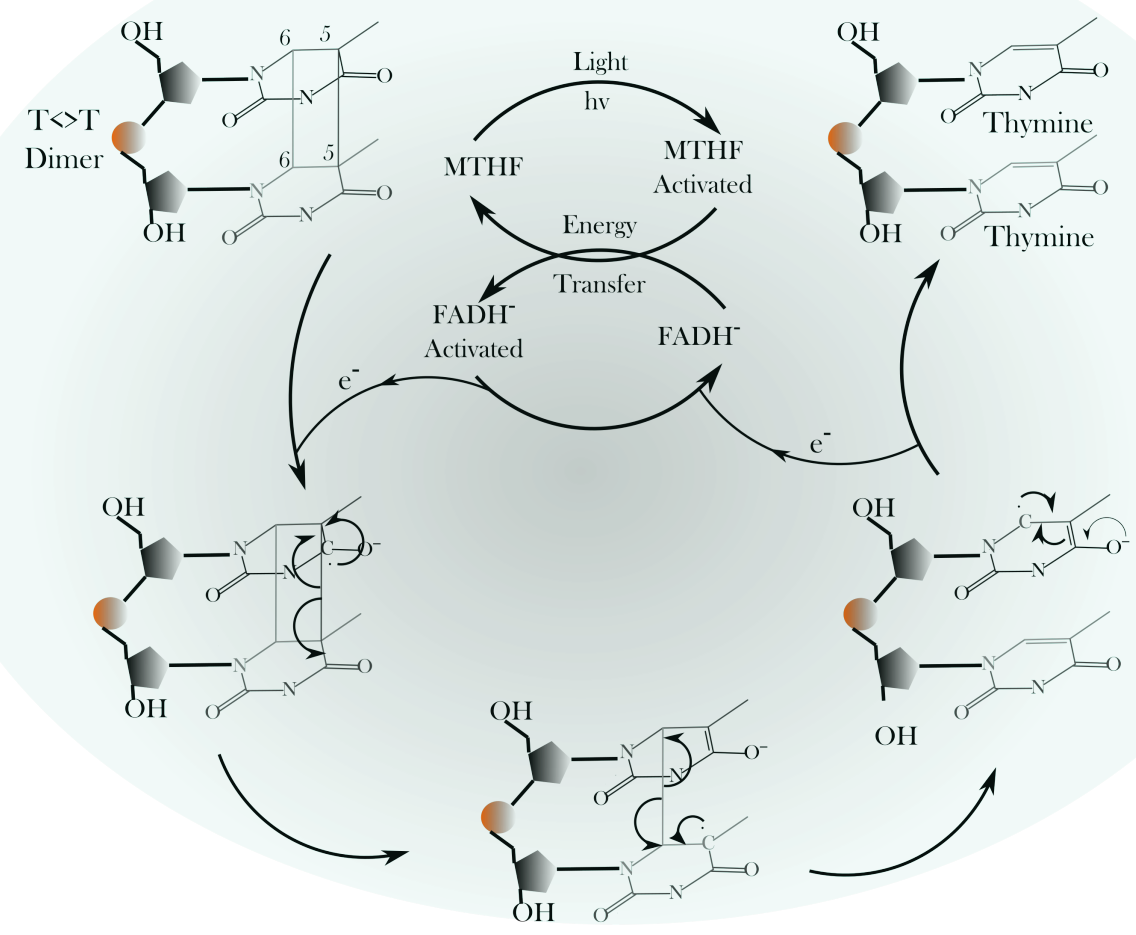

Fig. 7: Reaction mechanism of E.coli CPD photolyase. First, methenyltetrahydrofolate absorbs a photon from the blue light wavelengths and transfers the energy to flavin. The excited electron from Flavin (FAD) breaks the cyclobutane ring and is transferred back to flavin.

Studies with EcPL (Tan et al., 2014) and ssDNA repair enzyme of Vibrio cholerae CRY1 (Saxena, Wang, Kavakli, Sancar, \& Zhong, 2005) showed the rate of resonance energy transfer (RET) between the MTHF and $\mathrm{FADH}^{-}$in PLs depends on the distance between chromophores and their orientations within the enzyme. The RET time from $\mathrm{MTHF}^{*}$ was calculated to be $170 \mathrm{ps}$ for EcPL, where the distance between the two chromophores is exactly $16.8 \AA$. The RET time for VcCry1 where the distance between 
MTHF and $\mathrm{FADH}^{-}$is predicted as $15.2 \AA$ was calculated to be 60 ps (M. Zhang et al., 2017).

During the purification of the EcPL, where it is exposed to oxygen the FAD is found in neutral semiquinone form (FADH*) (Jorns, Sancar, \& Sancar, 1984). EnzymeFADH $^{*}$ is inactive unless it is treated with blue-light. Then energy is transferred to FADH $^{*}$ by nearby Trp residue within the EcPL. Trp306 is identified as a primary electron donor during the photoreduction of FAD (Y. F. Li, Heelis, \& Sancar, 1991). Then, in vitro studies proposed two electron transfer mechanisms from Trp306 to FADH*; electron hops "Trp triad", and electron travel through $\alpha$-helix side chain of Phe 366 . To understand whether such mechanism is part of the EcPL photocycle during the DNA repair in vivo mutant phr genes (Trp306Phe and Trp382Phe) are expressed at endogenous level in phr deficient E.coli. Results indicated that neither proposed electron transfer pathways are a part of the photocycle of EcPL (Kavakli \& Sancar, 2004).

\subsection{Reaction mechanisms of DNA repair mediated by (6-4) and other classes of the photolyases \\ Exposure of the DNA to UV irradiation for a long time not only generates Pyr $<$ Pyr but also 10-20\% of Pyr [6-4] Pyr photoproducts as well. Unlike Pyr $<>$ Pyr} photoproducts, Pyr [6-4] Pyr photoproducts are formed from the pyrimidine excited singlet state. Initially, Pyr [6-4] Pyr photoproduct is thought to be repaired by the excision repair system. An enzyme called (6-4) photolyase, which has the ability to directly repair Pyr [6-4] Pyr was discovered in Drosophila (Todo et al., 1993). Following that (6-4) photolyases were discovered in some other eukaryotic organisms. Therefore it is thought that this type of repair is only found in eukaryotic organisms. Recently, a new class has been discovered in more than 350 bacterial organisms (Oberpichler et al., 2011) 
and shown to possess Pyr [6-4] Pyr repair activity (F. Zhang et al., 2013). Despite the fact that (6-4) photolyases are very similar to CPD photolyases at the structural level and having a similar cofactor contents (Kim, Malhotra, Taylor, \& Sancar, 1996; Ozturk, 2017; Ozturk et al., 2008; Schelvis \& Gindt, 2017; Zhao et al., 1997), biochemical characterization of this enzyme revealed that (6-4) photolyases belong to different classes. Therefore, it is expected that class II, III, and (6-4) photolyases would have the same reaction mechanism as Class I photolyase. However, (6-4) photolyases must first thermally convert the open form of the (6-4) photoproducts to an oxetane intermediate before the repair which is the main difference to the CPD PLs. In contrast to CPD photoproducts, in which breaking of the UV light-induced bonds restores the bases to their canonical forms, the breaking of the $\mathrm{C}-5-\mathrm{OH}$ and $\mathrm{C} 6-\mathrm{C} 4$ bonds of the (6-4) photoproducts would not repair DNA but would in fact, generate two damaged bases. Therefore, formally an enzyme that repairs the (6-4) photoproduct must catalyze both bond breakage (lyase) and group transfer (transferase) reactions. Surprisingly, the (6-4) photolyase accomplishes this difficult task, even though it occurs with a rather low quantum yield (Hitomi et al., 1997). Several repair models were proposed for the (6-4) photolyase: i) oxetane intermediated formation in the ground state before photochemical reaction (Hitomi et al., 2001), ii) the primary photochemical reaction followed by water molecule formation to split bond breakage (Maul et al., 2008) and iii) a two-photon repair mechanism (Yamamoto, Plaza, \& Brettel, 2017). In any repair models, the involvement of two His residues, which are His354 and His 358 from Xenopus laevis (6-4) PL (X1PL), play critical roles (Hitomi et al., 2001) (His 365 and His 369 in Drosophila melanogaster PL, DmPL; His 364 and His 368 in Arabidopsis thaliana). The replacement of these 
amino acids results in significant activity loss of the X1(6-4) PL although the affinity of the mutants is comparable with the wild-type enzyme. In fact, X-ray crystallographic study indicates His354 (His 365 in DmPL) contacts with the OH group of C5 side of the $5^{\prime}$ (Fig. 8A) (Maul et al., 2008). The proton is transferred from the His354 (6-4) photoproducts to generate protonated neutral radical photoproduct for efficient DNA repair. 
A

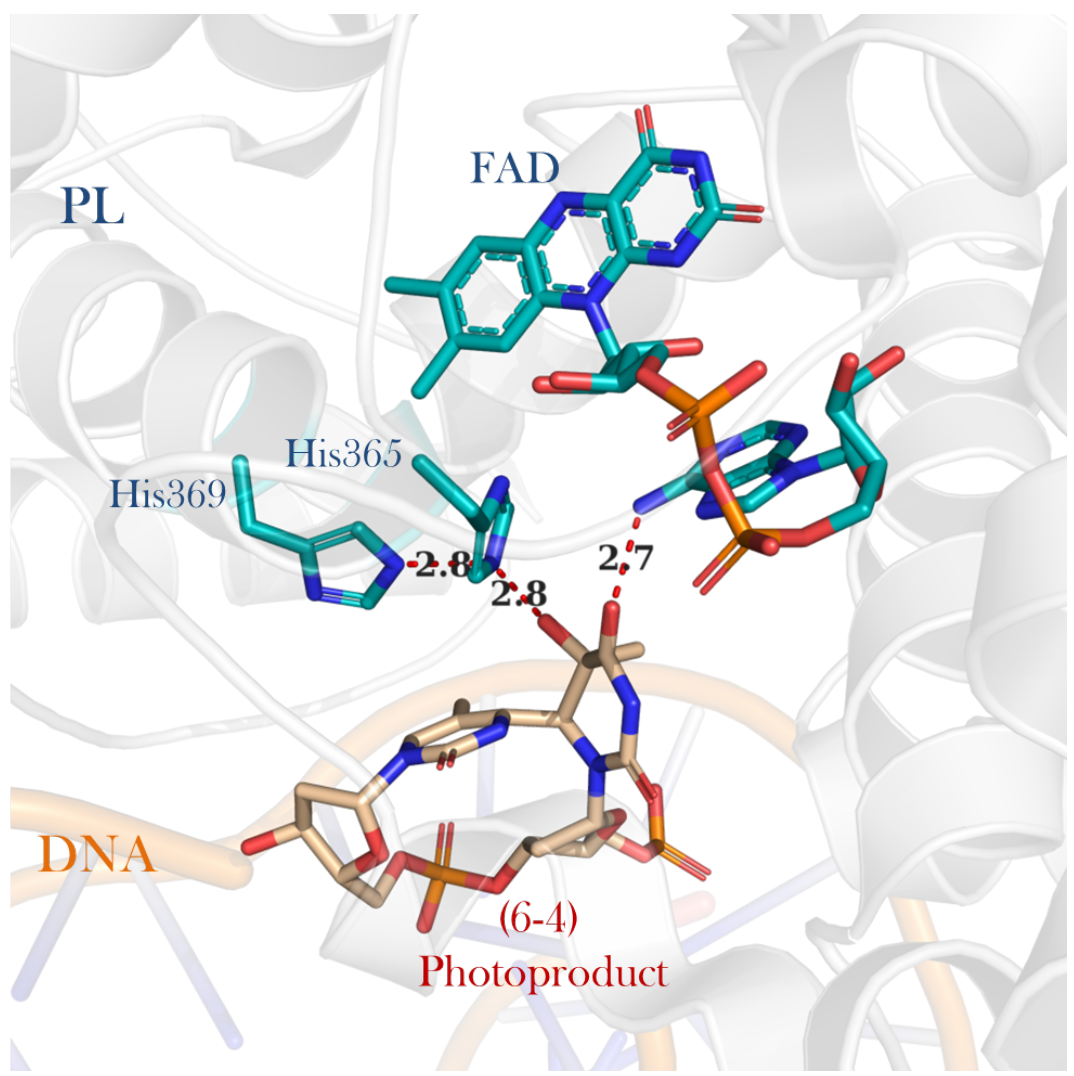

B

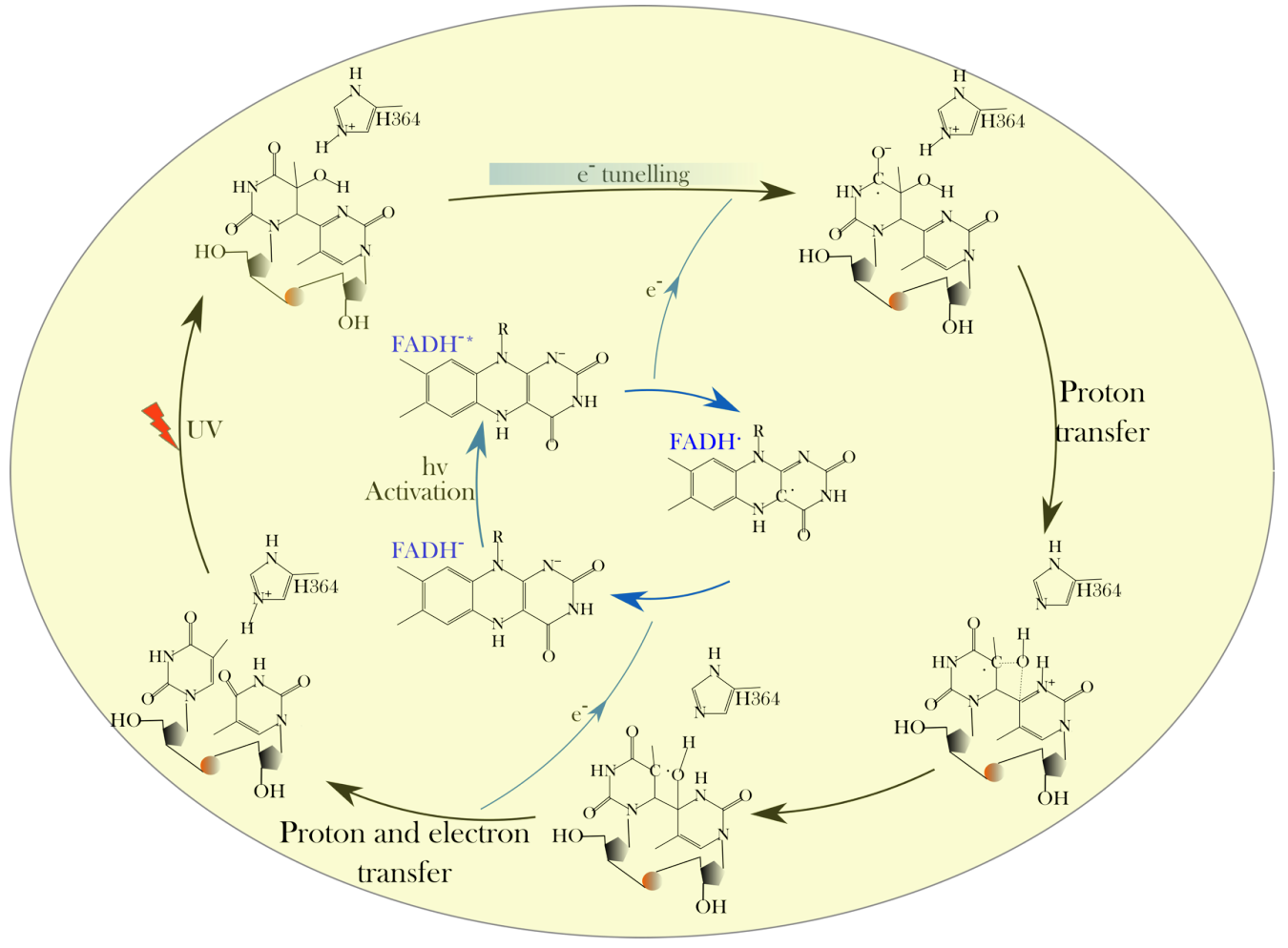


Fig. 8: A) Active site of the Drosophila melanogaster (6-4) photolyase (PDBID: 3CVU) with (6-4) photoproduct. During the course of catalysis, His365 donates a proton to repair (6-4) photoproduct. The red dotted line indicates the distance between the His residues to FAD in $\AA$. B)The photocycle DNA repair by (6-4) photolyase. In the very first step electron ejection occurs from substrate I to II in $280 \mathrm{ps}$ by direct electron tunneling from lumiflavin (LfH) to substrate via forward electron transfer (FET2). Then, a proton is transferred from His364 to substrate generating substrate III in 481 ps which is branched by the back electron transfer (BET2) occurring in 57 ps without any repair (from substrate II to substrate I). During the course of repair, several rearrangements occur within the substrate (from III to IV). Finally, the proton with an electron goes back His364 and generates two free thymine bases, which takes more than 10 ns. Reproduced from Zhang et al. (M. Zhang et al., 2017) with permission

By using the ultrafast spectroscopy Li et al (J. Li et al., 2010) showed the steps of the catalysis in At (6-4) PL. They revealed that the repair is a cyclic proton transfer between the enzyme and the substrate, where a single electron completely repairs the (64) photoproduct in its ground state. As shown in Fig. 8B, once photoinduced charge separation occurs between the FAD and the substrates, the reactions proceed in different pathways. These pathways have different backward and forward energies, which causes a quantum yield of DNA repair comparable to CPD photolyase. Additionally, they also showed proton transfer (His354 in Xl (6-4) PL) is the rate-limiting step in the catalysis of the (6-4) PL. It is expected that Class III and ssDNA photolyases would have the same reaction mechanism as Class I repair photolyases.

\section{Conclusion}

Phylogenetic analyses of cryptochrome/photolyase protein family have been revealed for different classes of the photolyases. Although homology of PLs is quite low at the primary structures level, the comparisons of the crystal structures of the PLs reveal 
remarkable similarity among different classes with some differences. Studies with enzyme-substrate co-crystal structures of different PLs and the identification of reaction intermediates by fast spectroscopic methods enabled us to pinpoint reaction mechanism during the course of DNA repair. Especially, recent studies highlighted CPD and (6-4) photolyases use a very similar energy transfer strategy with a cyclic electron transfer radical mechanism consistent with the in vivo studies. Exceptionally, a critical proton donation from His residue is needed to transfer oxygen atoms between two damaged bases during the course DNA repair mediated by (6-4) photolyases. Photolyase still remains an excellent system to study intra-protein energy transfer with multiple tunneling or hopping pathways.

\section{Acknowledgment}

We would like to thank Dr. Mehmet Tardu for helping us to generate Figure 2. We also like to thanks Anna Elms for her critical reading of the manuscript.

\section{References}

Asimgil, H., \& Kavakli, I. H. (2012). Purification and characterization of five members of photolyase/cryptochrome family from Cyanidioschyzon merolae. Plant Science

, 185-186, 190-198. doi:10.1016/j.plantsci.2011.10.005

Baer, M. E., \& Sancar, G. B. (1993). The role of conserved amino acids in substrate binding and discrimination by photolyase. J Biol Chem, 268(22), 1671716724.

Bais, A. F., McKenzie, R. L., Bernhard, G., Aucamp, P. J., Ilyas, M., Madronich, S., \& Tourpali, K. (2015). Ozone depletion and climate change: impacts on UV radiation. Photochem Photobiol Sci, 14(1), 19-52. doi:10.1039/c4pp90032d 
Brudler, R., Hitomi, K., Daiyasu, H., Toh, H., Kucho, K., Ishiura, M., . . G Getzoff, E. D. (2003). Identification of a new cryptochrome class. Structure, function, and evolution. Mol Cell, 11(1), 59-67.

Chaves, I., Pokorny, R., Byrdin, M., Hoang, N., Ritz, T., Brettel, K., ... Ahmad, M. (2011). The cryptochromes: blue light photoreceptors in plants and animals. Annu Rev Plant Biol, 62, 335-364. doi:10.1146/annurev-arplant-042110103759

Chaves, I., Yagita, K., Barnhoorn, S., Okamura, H., van der Horst, G. T. J., \& Tamanini, F. (2006). Functional evolution of the photolyase/cryptochrome protein family: Importance of the $C$ terminus of mammalian CRY1 for circadian core oscillator performance. Molecular and Cellular Biology, 26(5), 1743-1753. doi:Doi 10.1128/Mcb.26.5.1743-1753.2006

Eker, A. P., Yajima, H., \& Yasui, A. (1994). DNA photolyase from the fungus Neurospora crassa. Purification, characterization and comparison with other photolyases. Photochem Photobiol, 60(2), 125-133.

Fujihashi, M., Numoto, N., Kobayashi, Y., Mizushima, A., Tsujimura, M., Nakamura, A., ... Miki, K. (2007). Crystal structure of archaeal photolyase from Sulfolobus tokodaii with two FAD molecules: implication of a novel light-harvesting cofactor. J Mol Biol, 365(4), 903-910. doi:10.1016/j.jmb.2006.10.012

Graf, D., Wesslowski, J., Ma, H., Scheerer, P., Krauss, N., Oberpichler, I., . . L Lamparter, T. (2015). Key Amino Acids in the Bacterial (6-4) Photolyase PhrB from Agrobacterium fabrum. PLoS One, 10(10), e0140955. doi:10.1371/journal.pone.0140955

Harm, W., Harm.H., \& Rupert, C. S. (1968). Analysis of photoenzymatic repair of UV lesions in DNA by single light flashes. II. In vivo studies with Escherichia coli cells and bacteriophage. Mutat Res., 6(3), 371-385.

Hitomi, K., DiTacchio, L., Arvai, A. S., Yamamoto, J., Kim, S. T., Todo, T., . . . Getzoff, E. D. (2009). Functional motifs in the (6-4) photolyase crystal structure make a comparative framework for DNA repair photolyases and clock cryptochromes. Proceedings of the National Academy of Sciences of the United States of America, 106(17), 6962-6967. doi:10.1073/pnas.0809180106

Hitomi, K., Kim, S. T., Iwai, S., Harima, N., Otoshi, E., Ikenaga, M., \& Todo, T. (1997). Binding and catalytic properties of Xenopus (6-4) photolyase. J Biol Chem, 272(51), 32591-32598.

Hitomi, K., Nakamura, H., Kim, S. T., Mizukoshi, T., Ishikawa, T., Iwai, S., \& Todo, T. (2001). Role of two histidines in the (6-4) photolyase reaction. J Biol Chem, 276(13), 10103-10109. doi:10.1074/jbc.M008828200

Hitomi, K., Okamoto, K., Daiyasu, H., Miyashita, H., Iwai, S., Toh, H., ... Todo, T. (2000). Bacterial cryptochrome and photolyase: characterization of two photolyase-like genes of Synechocystis sp. PCC6803. Nucleic Acids Res, 28(12), 2353-2362.

Huang, Y., Baxter, R., Smith, B. S., Partch, C. L., Colbert, C. L., \& Deisenhofer, J. (2006). Crystal structure of cryptochrome 3 from Arabidopsis thaliana and its implications for photolyase activity. Proc Natl Acad Sci U S A, 103(47), 1770117706. doi:10.1073/pnas.0608554103 
Husain, I., \& Sancar, A. (1987). Binding of E. coli DNA photolyase to a defined substrate containing a single T mean value of T dimer. Nucleic Acids Res, 15(3), 1109-1120.

Jordan, S. P., Alderfer, J. L., Chanderkar, L. P., \& Jorns, M. S. (1989). Reaction of Escherichia coli and yeast photolyases with homogeneous short-chain oligonucleotide substrates. Biochemistry, 28(20), 8149-8153.

Jorns, M. S., Baldwin, E. T., Sancar, G. B., \& Sancar, A. (1987). Action mechanism of Escherichia coli DNA photolyase. II. Role of the chromophores in catalysis. $J$ Biol Chem, 262(1), 486-491.

Jorns, M. S., Sancar, G. B., \& Sancar, A. (1984). Identification of a neutral flavin radical and characterization of a second chromophore in Escherichia coli DNA photolyase. Biochemistry, 23(12), 2673-2679.

Jorns, M. S., Sancar, G. B., \& Sancar, A. (1985). Identification of Oligothymidylates as New Simple Substrates for Escherichia-Coli DNA Photolyase and Their Use in a Rapid Spectrophotometric Enzyme Assay. Biochemistry, 24(8), 1856-1861. doi:DOI 10.1021/bi00329a008

Kavakli, I. H., Baris, I., Tardu, M., Gul, S., Oner, H., Cal, S., .. A Aydin, C. (2017). The Photolyase/Cryptochrome Family of Proteins as DNA Repair Enzymes and Transcriptional Repressors. Photochem Photobiol, 93(1), 93-103. doi:10.1111/php.12669

Kavakli, I. H., \& Sancar, A. (2004). Analysis of the role of intraprotein electron transfer in photoreactivation by DNA photolyase in vivo. Biochemistry, 43(48), 15103-15110. doi:10.1021/bi0478796

Kim, S. T., Malhotra, K., Taylor, J. S., \& Sancar, A. (1996). Purification and partial characterization of (6-4) photoproduct DNA photolyase from Xenopus laevis. Photochem Photobiol, 63(3), 292-295.

Kim, S. T., Sancar, A., Essenmacher, C., \& Babcock, G. T. (1993). Time-Resolved Epr Studies with DNA Photolyase - Excited-State Fadh(0) Abstracts an Electron from Trp-306 to Generate Fadh-, the Catalytically Active Form of the Cofactor. Proceedings of the National Academy of Sciences of the United States of America, 90(17), 8023-8027. doi:DOI 10.1073/pnas.90.17.8023

Kiontke, S., Geisselbrecht, Y., Pokorny, R., Carell, T., Batschauer, A., \& Essen, L. 0. (2011). Crystal structures of an archaeal class II DNA photolyase and its complex with UV-damaged duplex DNA. Embo Journal, 30(21), 4437-4449. doi:10.1038/emboj.2011.313

Kizilel, R., Demir, E., Azizoglu, S., Asimgil, H., Kavakli, I. H., \& Kizilel, S. (2012). Investigation of real-time photorepair activity on DNA via surface plasmon resonance. PLoS One, 7(8), e44392. doi:10.1371/journal.pone.0044392

Klar, T., Pokorny, R., Moldt, J., Batschauer, A., \& Essen, L. O. (2007). Cryptochrome 3 from Arabidopsis thaliana: structural and functional analysis of its complex with a folate light antenna. J Mol Biol, 366(3), 954-964.

doi:10.1016/j.jmb.2006.11.066

Komori, H., Masui, R., Kuramitsu, S., Yokoyama, S., Shibata, T., Inoue, Y., \& Miki, K. (2001). Crystal structure of thermostable DNA photolyase: pyrimidine-dimer recognition mechanism. Proc Natl Acad Sci U S A, 98(24), 13560-13565. doi:10.1073/pnas.241371398 
Konig, S., Juhas, M., Jager, S., Kottke, T., \& Buchel, C. (2017). The cryptochromephotolyase protein family in diatoms. J Plant Physiol, 217, 15-19. doi:10.1016/j.jplph.2017.06.015

Li, J., Liu, Z., Tan, C., Guo, X., Wang, L., Sancar, A., \& Zhong, D. (2010). Dynamics and mechanism of repair of ultraviolet-induced (6-4) photoproduct by photolyase. Nature, 466(7308), 887-890. doi:10.1038/nature09192

Li, Y. F., Heelis, P. F., \& Sancar, A. (1991). Active site of DNA photolyase: tryptophan306 is the intrinsic hydrogen atom donor essential for flavin radical photoreduction and DNA repair in vitro. Biochemistry, 30(25), 6322-6329.

Liu, Z., Tan, C., Guo, X., Kao, Y. T., Li, J., Wang, L., ... Zhong, D. (2011). Dynamics and mechanism of cyclobutane pyrimidine dimer repair by DNA photolyase. Proc Natl Acad Sci U S A, 108(36), 14831-14836. doi:10.1073/pnas.1110927108

Liu, Z., Zhang, M., Guo, X., Tan, C., Li, J., Wang, L., ... Zhong, D. (2013). Dynamic determination of the functional state in photolyase and the implication for cryptochrome. Proc Natl Acad Sci U S A, 110(32), 12972-12977. doi:10.1073/pnas.1311077110

Maul, M. J., Barends, T. R., Glas, A. F., Cryle, M. J., Domratcheva, T., Schneider, S., ... Carell, T. (2008). Crystal structure and mechanism of a DNA (6-4) photolyase. Angew Chem Int Ed Engl, 47(52), 10076-10080. doi:10.1002/anie.200804268

Mees, A., Klar, T., Gnau, P., Hennecke, U., Eker, A. P. M., Carell, T., \& Essen, L. 0. (2004). Crystal structure of a photolyase bound to a CPD-like DNA lesion after in situ repair. Science, 306(5702), 1789-1793. doi:DOI 10.1126/science.1101598

Menck, C. F. (2002). Shining a light on photolyases. Nat Genet, 32(3), 338-339. doi:10.1038/ng1102-338

Michael, A. K., Fribourgh, J. L., Van Gelder, R. N., \& Partch, C. L. (2017). Animal Cryptochromes: Divergent Roles in Light Perception, Circadian Timekeeping and Beyond. Photochem Photobiol, 93(1), 128-140. doi:10.1111/php.12677

Noordally, Z. B., \& Millar, A. J. (2015). Clocks in algae. Biochemistry, 54(2), 171-183. doi:10.1021/bi501089x

Oberpichler, I., Pierik, A. J., Wesslowski, J., Pokorny, R., Rosen, R., Vugman, M., . . . Lamparter, T. (2011). A photolyase-like protein from Agrobacterium tumefaciens with an iron-sulfur cluster. PLoS One, 6(10), e26775. doi:10.1371/journal.pone.0026775

Ozturk, N. (2017). Phylogenetic and Functional Classification of the Photolyase/Cryptochrome Family. Photochem Photobiol, 93(1), 104-111. doi:10.1111/php.12676

Ozturk, N., Kao, Y. T., Selby, C. P., Kavakli, I. H., Partch, C. L., Zhong, D., \& Sancar, A. (2008). Purification and characterization of a type III photolyase from Caulobacter crescentus. Biochemistry, 47(39), 10255-10261. doi:10.1021/bi801085a

Ozturk, N., Song, S. H., Ozgur, S., Selby, C. P., Morrison, L., Partch, C., . . . Sancar, A. (2007). Structure and function of animal cryptochromes. Cold Spring Harb Symp Quant Biol, 72, 119-131. doi:10.1101/sqb.2007.72.015

Park, H. W., Kim, S. T., Sancar, A., \& Deisenhofer, J. (1995). Crystal structure of DNA photolyase from Escherichia coli. Science, 268(5219), 1866-1872. 
Payne, G., \& Sancar, A. (1990). Absolute action spectrum of E-FADH2 and E-FADH2MTHF forms of Escherichia coli DNA photolyase. Biochemistry, 29(33), 77157727.

Sancar, A. (2003). Structure and function of DNA photolyase and cryptochrome blue-light photoreceptors. Chemical Reviews, 103(6), 2203-2237. doi:10.1021/cr0204348

Sancar, A. (2008). Structure and function of photolyase and in vivo enzymology: 50th anniversary. J Biol Chem, 283(47), 32153-32157. doi:10.1074/jbc.R800052200

Sancar, A. (2016). Mechanisms of DNA Repair by Photolyase and Excision Nuclease (Nobel Lecture). Angew Chem Int Ed Engl, 55(30), 8502-8527. doi:10.1002/anie.201601524

Sancar, A., \& Sancar, G. B. (1984). Escherichia-Coli DNA Photolyase Is a Flavoprotein. Journal of Molecular Biology, 172(2), 223-227. doi:Doi 10.1016/S00222836(84)80040-6

Sancar, G. B., \& Sancar, A. (1987). Structure and Function of DNA Photolyases. Trends in Biochemical Sciences, 12(7), 259-261. doi:Doi 10.1016/09680004(87)90130-7

Sancar, G. B., Smith, F. W., Reid, R., Payne, G., Levy, M., \& Sancar, A. (1987). Action mechanism of Escherichia coli DNA photolyase. I. Formation of the enzymesubstrate complex. J Biol Chem, 262(1), 478-485.

Saxena, C., Wang, H., Kavakli, I. H., Sancar, A., \& Zhong, D. (2005). Ultrafast dynamics of resonance energy transfer in cryptochrome. J Am Chem Soc, 127(22), 79847985. doi:10.1021/ja0421607

Scheerer, P., Zhang, F., Kalms, J., von Stetten, D., Krauss, N., Oberpichler, I., \& Lamparter, T. (2015). The class III cyclobutane pyrimidine dimer photolyase structure reveals a new antenna chromophore binding site and alternative photoreduction pathways. J Biol Chem, 290(18), 11504-11514. doi:10.1074/jbc.M115.637868

Schelvis, J. P., \& Gindt, Y. M. (2017). A Review of Spectroscopic and BiophysicalChemical Studies of the Complex of Cyclobutane Pyrimidine Dimer Photolyase and Cryptochrome DASH with Substrate DNA. Photochem Photobiol, 93(1), 26-36. doi:10.1111/php.12678

Selby, C. P., \& Sancar, A. (2006). A cryptochrome/photolyase class of enzymes with single-stranded DNA-specific photolyase activity. Proc Natl Acad Sci U S A, 103(47), 17696-17700. doi:10.1073/pnas.0607993103

Tamada, T., Kitadokoro, K., Higuchi, Y., Inaka, K., Yasui, A., de Ruiter, P. E., . . . Miki, K. (1997). Crystal structure of DNA photolyase from Anacystis nidulans. Nat Struct Biol, 4(11), 887-891.

Tan, C., Guo, L., Ai, Y., Li, J., Wang, L., Sancar, A., ... Zhong, D. (2014). Direct determination of resonance energy transfer in photolyase: structural alignment for the functional state. J Phys Chem A, 118(45), 10522-10530. doi:10.1021/jp504349b

Tan, C., Liu, Z., Li, J., Guo, X., Wang, L., Sancar, A., \& Zhong, D. (2015). The molecular origin of high DNA-repair efficiency by photolyase. Nat Commun, 6, 7302. doi:10.1038/ncomms8302 
Thompson, C. L., \& Sancar, A. (2002). Photolyase/cryptochrome blue-light photoreceptors use photon energy to repair DNA and reset the circadian clock. Oncogene, 21(58), 9043-9056. doi:10.1038/sj.onc.1205958

Todo, T., Takemori, H., Ryo, H., Ihara, M., Matsunaga, T., Nikaido, O., ... Nomura, T. (1993). A New Photoreactivating Enzyme That Specifically Repairs Ultraviolet Light-Induced (6-4)Photoproducts. Nature, 361(6410), 371-374. doi:DOI 10.1038/361371a0

Ueda, T., Kato, A., Kuramitsu, S., Terasawa, H., \& Shimada, I. (2005). Identification and characterization of a second chromophore of DNA photolyase from Thermus thermophilus HB27. J Biol Chem, 280(43), 36237-36243. doi:10.1074/jbc.M507972200

White, M. F., \& Dillingham, M. S. (2012). Iron-sulphur clusters in nucleic acid processing enzymes. Current Opinion in Structural Biology, 22(1), 94-100. doi:10.1016/j.sbi.2011.11.004

Worthington, E. N., Kavakli, I. H., Berrocal-Tito, G., Bondo, B. E., \& Sancar, A. (2003). Purification and characterization of three members of the photolyase/cryptochrome family blue-light photoreceptors from Vibrio cholerae. J Biol Chem, 278(40), 39143-39154. doi:10.1074/jbc.M305792200

Xu, L., Mu, W. M., Ding, Y. W., Luo, Z. F., Han, Q. K., Bi, F. Y., ... Song, Q. H. (2008). Active site of Escherichia coli DNA photolyase: Asn378 is crucial both for stabilizing the neutral flavin radical cofactor and for DNA repair. Biochemistry, 47(33), 8736-8743. doi:10.1021/bi800391j

Yamamoto, J., Plaza, P., \& Brettel, K. (2017). Repair of (6-4) Lesions in DNA by (6-4) Photolyase: 20 Years of Quest for the Photoreaction Mechanism. Photochem Photobiol, 93(1), 51-66. doi:10.1111/php.12696

Yi, C., \& He, C. (2013). DNA repair by reversal of DNA damage. Cold Spring Harb Perspect Biol, 5(1), a012575. doi:10.1101/cshperspect.a012575

Zhang, F., Ma, H., Bowatte, K., Kwiatkowski, D., Mittmann, E., Qasem, H., . . . Lamparter, T. (2017). Crystal Structures of Bacterial (6-4) Photolyase Mutants with Impaired DNA Repair Activity. Photochem Photobiol, 93(1), 304-314. doi:10.1111/php.12699

Zhang, F., Scheerer, P., Oberpichler, I., Lamparter, T., \& Krauss, N. (2013). Crystal structure of a prokaryotic (6-4) photolyase with an Fe-S cluster and a 6,7dimethyl-8-ribityllumazine antenna chromophore. Proc Natl Acad Sci U S A, 110(18), 7217-7222. doi:10.1073/pnas.1302377110

Zhang, M., Wang, L., \& Zhong, D. (2017). Photolyase: Dynamics and electron-transfer mechanisms of DNA repair. Arch Biochem Biophys, 632, 158-174. doi:10.1016/j.abb.2017.08.007

Zhao, X., Liu, J., Hsu, D. S., Zhao, S., Taylor, J. S., \& Sancar, A. (1997). Reaction mechanism of (6-4) photolyase. J Biol Chem, 272(51), 32580-32590. 